\title{
Real-World Clinical Application of 12-Week Sofosbuvir/Velpatasvir Treatment for Decompensated Cirrhotic Patients with Genotype 1 and 2: A Prospective, Multicenter Study
}

\begin{abstract}
Masanori Atsukawa · Akihito Tsubota · Chisa Kondo $\cdot$ Hidenori Toyoda $\cdot$ Makoto Nakamuta $\cdot$ Koichi Takaguchi Tsunamasa Watanabe - Atsushi Hiraoka - Haruki Uojima - Toru Ishikawa - Motoh Iwasa · Toshifumi Tada • Akito Nozaki · Makoto Chuma - Shinya Fukunishi · Akira Asai · Toru Asano · Chikara Ogawa · Hiroshi Abe • Naoki Hotta · Toshihide Shima • Etsuko Iio · Shigeru Mikami · Yoshihiko Tachi · Shinichi Fujioka • Hironao Okubo · Noritomo Shimada · Joji Tani • Isao Hidaka • Akio Moriya • Kunihiko Tsuji · Takehiro Akahane Naoki Yamashita - Tomomi Okubo - Taeang Arai - Kiyoshi Morita - Kazuhito Kawata • Yasuhito Tanaka • Takeshi Okanoue $\cdot$ Shin Maeda · Takashi Kumada $\cdot$ Katsuhiko Iwakiri on behalf of KTK49 Liver Study Group
\end{abstract}

Received: July 11, 2020 / Published online: September 8, 2020

(C) The Author(s) 2020

\section{ABSTRACT}

Introduction: Clinical trials of direct-acting antivirals for patients with decompensated

Digital Features To view digital features for this article go to https://doi.org/10.6084/m9.figshare.12770987.

The members of KTK49 Liver Study Group are listed in Acknowledgements.

Akihito Tsubota and Masanori Atsukawa contributed equally to the preparation of this manuscript.

Electronic supplementary material The online version of this article (https://doi.org/10.1007/s40121020-00329-y) contains supplementary material, which is available to authorized users.

M. Atsukawa $(\varangle) \cdot$ C. Kondo · T. Okubo · T. Arai · K. Iwakiri

Division of Gastroenterology and Hepatology, Department of Internal Medicine, Nippon Medical School, Tokyo, Japan

e-mail: momogachi@yahoo.co.jp

A. Tsubota $(\bowtie)$

Core Research Facilities for Basic Science, The Jikei University School of Medicine, Tokyo, Japan e-mail: atsubo@jikei.ac.jp cirrhosis have been conducted, but there is limited information on the medicinal applications in clinical settings. We aimed to evaluate the safety and efficacy of sofosbuvir/velpatasvir for decompensated cirrhotic patients with genotypes 1 and 2 in real-world clinical practice.

Methods: A prospective, multicenter study of 12-week sofosbuvir/velpatasvir was conducted for patients with decompensated cirrhosis at 33 institutions.

Results: The cohort included 71 patients (52 genotype 1, 19 genotype 2): 7 with Child-Pugh class A, 47 with class B, and 17 with class C (median score 8; range 5-13). The albumin-bilirubin (ALBI) score ranged from - 3.01 to -0.45 (median -1.58 ). Sixty-nine patients

H. Toyoda · T. Kumada

Department of Gastroenterology, Ogaki Municipal Hospital, Ogaki, Gifu, Japan

\section{Nakamuta $\cdot$ N. Yamashita}

National Hospital Organization Kyushu Medical

Center, Fukuoka, Japan

K. Takaguchi

Department of Hepatology, Kagawa Prefectural

Central Hospital, Takamatsu, Kagawa, Japan 
(97.2\%) completed treatment as scheduled. The overall rate of sustained virologic response at 12 weeks post-treatment (SVR12) was 94.4\% (67/71). SVR12 rates in the patients with Child-Pugh classes A, B, and C were $85.7 \%$, $97.9 \%$, and $88.2 \%$, respectively. Among 22 patients with a history of hepatocellular carcinoma treatment, 20 (90.9\%) achieved SVR12. The Child-Pugh score and ALBI grade significantly improved after achieving SVR12 $\left(p=7.19 \times 10^{-4}\right.$ and $2.42 \times 10^{-4}$, respectively) . Notably, the use of diuretics and branchedchain amino acid preparations significantly reduced after achieving SVR12. Adverse events were observed in $19.7 \%$ of the patients, leading to treatment discontinuation in two patients with cholecystitis and esophageal varices rupture, respectively.

T. Watanabe

Department of Internal Medicine, St. Marianna

University School of Medicine, Kawasaki, Kanagawa, Japan

\section{A. Hiraoka}

Gastroenterology Center, Ehime Prefectural Central Hospital, Matsuyama, Ehime, Japan

H. Uojima

Department of Gastroenterology, Internal Medicine, Kitasato University School of Medicine, Kanagawa, Japan

T. Ishikawa

Department of Hepatology, Saiseikai Niigata

Hospital, Niigata, Japan

M. Iwasa

Department of Gastroenterology and Hepatology,

Mie University School of Medicine, Mie, Japan

T. Tada

Department of Gastroenterology, Himeji Red Cross

Hospital, Hyogo, Japan

A. Nozaki $\cdot$ M. Chuma $\cdot$ S. Maeda

Gastroenterological Center, Yokohama City

University Medical Center, Yokohama, Japan
Conclusion: Twelve weeks of sofosbuvir/velpatasvir in real-world clinical practice yielded high SVR rates and acceptable safety profiles in decompensated cirrhotic patients with genotypes 1 and 2. Achievement of SVR not only restored the liver functional reserve but also reduced or spared the administration of drugs for related complications.

Trial Registration: UMIN registration no, 000038587.

Keywords: ALBI grade; Child-Pugh class; Decompensated cirrhosis; Hepatitis C virus; Sofosbuvir; Velpatasvir
S. Fukunishi · A. Asai

Second Department of Internal Medicine, Osaka Medical College, Osaka, Japan

\section{T. Asano}

Division of Gastroenterology and Hepatology, Department of Internal Medicine, Tokyo Metropolitan Bokutoh Hospital, Tokyo, Japan

\section{Ogawa}

Department of Gastroenterology and Hepatology, Takamatsu Red Cross Hospital, Takamatsu, Japan

H. Abe

Division of Gastroenterology and Hepatology, Department of Internal Medicine, Shinmatusdo Central General Hospital, Matsudo, Japan

\section{N. Hotta}

Division of Hepatology, Department of Internal Medicine, Masuko Memorial Hospital, Nagoya, Japan

T. Shima - T. Okanoue Department of Gastroenterology and Hepatology, Saiseikai Suita Hospital, Suita, Japan 


\section{Key Summary Points}

\section{Why carry out this study?}

Real-world patient cohorts may include patients with unfavorable factors or conditions, unlike the participants in phase 3 clinical trials.

To confirm the real-world efficacy and safety of 12-week sofosbuvir/velpatasvir (SOF/VEL) (without ribavirin), we performed a prospective multicenter study of decompensated cirrhotic patients, including those who would be ineligible for clinical trials, and addressed how sustained virologic response (SVR) achievement could have a beneficial impact on such patients within a short period in real-world clinical practice.

Among 71 patients, 69 (97.2\%) completed treatment as scheduled, $71(100 \%)$ had virological response at the end (or cessation) of treatment, and 67 (94.4\%) achieved sustained virologic response at 12 weeks post-treatment (SVR12).
E. Iio $\cdot$ Y. Tanaka

Department of Virology and Liver Unit, Graduate School of Medical Sciences, Nagoya City University, Nagoya, Aichi, Japan

S. Mikami

Division of Gastroenterology, Department of Internal Medicine, Kikkoman General Hospital, Noda, Japan

\section{Y. Tachi}

Bantane Hospital, Fujita Health University School of Medicine, Nagoya, Japan

S. Fujioka

Department of Gastroenterology, Okayama Saiseikai General Hospital, Okayama, Japan

H. Okubo

Department of Gastroenterology, Juntendo Nerima University Hospital, Tokyo, Japan

N. Shimada

Division of Gastroenterology and Hepatology, Department of Internal Medicine, Otakanomori Hospital, Kashiwa, Japan
Twelve weeks of SOF/VEL yielded high efficacy, acceptable safety, and good tolerability, even in patients exhibiting advanced disease stages or unfavorable conditions. The achievement of SVR not only restored the liver functional reserve but also reduced or spared the administration of drugs for related complications.

\section{What was learned from the study?}

In real-world clinical practice, achieving SVR with SOF/VEL not only restored the liver functional reserve but also reduced or spared the use of diuretics and branchedchain amino acid preparations for the treatment of cirrhosis-related complications in the short term.

These findings suggest that direct-acting antiviral treatment such as SOF/VEL may improve health-related quality of life and prognosis and reduce physical and economic burdens of decompensated cirrhotic patients.
J. Tani

Department of Gastroenterology, Kagawa University Graduate School of Medicine, Kita-gun, Kagawa, Japan

\section{Hidaka}

Department of Gastroenterology and Hepatology, Yamaguchi University Graduate School of Medicine, Ube, Japan

\author{
A. Moriya \\ Department of Gastroenterology, Mitoyo General \\ Hospital, Kannonji, Japan
}

\section{K. Tsuji}

Gastroenterology Center, Teine Keijinkai Hospital, Sapporo, Japan

\section{T. Akahane}

Department of Gastroenterology, Japanese Red Cross Ishinomaki Hospital, Ishinomaki, Japan

\section{K. Morita}

Gastroenterology, Toyota Kosei Hospital, Toyota, Japan 


\section{DIGITAL FEATURES}

This article is published with digital features to facilitate understanding of the article. You can access the digital features on the article's associated Figshare page. To view digital features for this article go to https://doi.org/10.6084/m9. figshare.12770987.

\section{BACKGROUND}

Chronic hepatitis C virus (HCV) infection causes hepatic necroinflammation and fibrogenesis, and, consequently, is a primary risk factor for liver cirrhosis and hepatocellular carcinoma (HCC). Liver fibrosis gradually progresses through chronic active hepatitis to compensated cirrhosis, and eventually to decompensated cirrhosis, which is accompanied by various portal hypertension-related complications, such as ascites, gastrointestinal hemorrhage, and hepatic encephalopathy [1]. A recent meta-analysis showed that the 2-year survival rates of patients with Child-Pugh classes B and C were $70 \%$ and $40 \%$, respectively [1]. A Spanish cohort study reported that the mean survival of patients with decompensated cirrhosis was 4.1 years [2]. Therefore, a breakthrough treatment is urgently desired in order to improve the poor prognosis of decompensated cirrhosis.

Currently, direct-acting antiviral agents (DAAs) are the first-line standard of care for chronic $\mathrm{HCV}$ infection. We have previously reported that HCV elimination was achieved in 95-99\% of patients with chronic hepatitis or compensated cirrhosis [3-10], although our reports excluded patients with decompensated cirrhosis. Meanwhile, several real-world studies from Europe and the United States have included decompensated cirrhotic patients, but only

\section{K. Kawata}

Hepatology Division, Department of Internal Medicine II, Hamamatsu University School of Medicine, Hamamatsu, Japan

K. Kawata

KTK49 Liver Study Group, Kanto-Tokai-ShikokuKyushu, Japan one phase 3 study focusing on decompensated cirrhotic patients has been reported in Asia, by Japan [11-17]. Considering that patients with hepatitis $C$ in Japan are generally older than those in Europe and the United States [3-17], it is necessary to evaluate the efficacy and safety of DAAs for decompensated cirrhotic patients in real-world clinical practice in Japan.

DAAs available for treating HCV include NS3/ $4 \mathrm{~A}$ protease inhibitors, NS5A inhibitors, and NS5B polymerase inhibitors. Generally, two or more of these different drugs are administered in combination to treat chronic $\mathrm{HCV}$ infection. However, given the hepatotoxicity of some NS3/ $4 \mathrm{~A}$ protease inhibitors $[4-10,18]$, the guidelines of the European Association for the Study of Liver and the American Association for the Study of Liver Diseases recommend combining an NS5A inhibitor and an NS5B polymerase inhibitor for patients with decompensated cirrhosis $[19,20]$. Indeed, clinical trials in Europe and the United States indicate that this combination yielded high sustained virologic response (SVR) rates and that the liver functional reserve improved after SVR achievement [11-14]. There have been only two phase 3 clinical studies of sofosbuvir/velpatasvir (SOF/VEL) with or without ribavirin in patients with decompensated cirrhosis [Child-Pugh class B (score of 7-9) in ASTRAL-4 or Child-Pugh score of $\leq 12$ in a Japanese phase 3 study] [13, 21]. The Japanese phase 3 study reported that the addition of ribavirin did not improve the SVR rate, but rather increased the frequency and severity of adverse events (AEs) [21]. Therefore, the 12-week SOF/ VEL regimen (without ribavirin) was approved in 2019 in Japan for patients with decompensated cirrhosis.

In this prospective, multicenter study, we aimed to evaluate the safety and efficacy of 12-week SOF/VEL (without ribavirin) for patients with decompensated cirrhosis, including those who would be ineligible for clinical trial settings (primary objective). In addition, we assessed how SVR achievement could have beneficial impacts, such as restoration of liver functional reserve and reduction in drug doses for cirrhosis-related complications, within a short period in real-world clinical practice (secondary objective). 


\section{METHODS}

\section{Patients}

This was a prospective, multicenter $(n=33)$ study conducted by the KTK49 Liver Study Group in Japan (UMIN registration no. 000038587). Decompensated cirrhotic patients with genotypes 1 and 2 were recruited and received 12-week SOF/VEL between March 2019 and December 2019. Patients consented to participate in the study and met the following inclusion criteria: (1) serum HCV ribonucleic acid (RNA) levels > $1.2 \log \mathrm{IU} / \mathrm{mL}$; (2) 18 years or over; and (3) decompensated liver cirrhosis (Child-Pugh classes B or C) at the time of entry into the study. The exclusion criteria were: (1) malignant tumors; (2) co-infection with human immunodeficiency virus or hepatitis B virus; (3) contraindicated concomitant drugs against SOF/VEL; and (4) estimated glomerular filtration rate (eGFR) of $<30 \mathrm{~mL} / \mathrm{min} / 1.73 \mathrm{~m}^{2}$. This study was approved by the ethics committee of Nippon Medical School (IRB \#R1-05-1129) and each institution. This study was performed in accordance with the 2013 Helsinki Declaration. All patients consented to provide their data. Written informed consent was obtained from each patient before enrollment.

\section{Treatment Protocol and Laboratory Tests}

Patients received one tablet of EPUCLUSA ${ }^{\circledR}$ per os once daily (400 mg SOF and $100 \mathrm{mg}$ VEL; Gilead Sciences, Foster City, CA, USA) for 12 weeks. Physical, hematological, and biochemical examinations were performed at treatment initiation, and every 4 weeks during the treatment and follow-up periods.

AEs including laboratory abnormalities were graded according to the Common Terminology Criteria for Adverse Events v.5.0, as presented by the National Cancer Institute Cancer Therapy Evaluation Program [22].

Serum HCV RNA levels were measured using a real-time polymerase chain reaction (PCR)based method (COBAS TaqMan HCV Test 2.0; Roche Molecular Systems, Pleasanton, CA, USA). The lower limit of quantification was 1.2 $\log \mathrm{IU} / \mathrm{mL}$. HCV genotype was determined using PCR with genotype-specific primers for amplifying the core gene sequence [23].

\section{Definition of Treatment Responses}

SVR12 was defined as undetectable serum HCV RNA at 12 weeks post-treatment. Patients with undetectable serum HCV RNA at the end of treatment were considered to have achieved the end-of-treatment response (EOT). Patients with EOT in whom viral RNA reappeared after treatment completion were considered to have relapsed.

\section{Evaluation of the Resistance-Associated Substitutions}

NS5A substitutions were determined using direct sequencing techniques. Extracted RNA samples from the serum were reverse-transcribed and amplified via the nested PCR method, using outer primers for the NS5A region: 5'-ATGAACC GRCTGATAGCGTT-3' [NS5A H-F1; sense, nucleotide (nt) 6075-6094, numbered according to HCV-J (accession no. D90208)] and 5'-CTA GCTGAAGAGCTGGCCAA-3' (NS5AH-R1; antisense, nt 6969-6988), and inner primers: $5^{\prime}-\mathrm{T}$ CCCCYACRCACTATGTGCC-3' (NS5A H-F2; sense, nt6117-6136) and 5'-CGCTTYGCCG TRTCTGCTGT-3' (NS5A H-R2; antisense, nt 6921-6940). The conditions of the first and second PCR rounds were the same: $95^{\circ} \mathrm{C}$ for $2 \mathrm{~min}$, followed by 40 cycles at $98^{\circ} \mathrm{C}$ for $10 \mathrm{~s}, 55^{\circ} \mathrm{C}$ for $15 \mathrm{~s}$, and $68^{\circ} \mathrm{C}$ for $1 \mathrm{~min}$. The PCR products were then sequenced on an automated DNA sequencer (3730xl DNA Analyzer; Thermo Fisher Scientific, Waltham, MA, USA). The resistanceassociated amino acid positions were referenced as in previous reports [24]. Subtype-specific reference strains were Con1 (AJ 238799) for genotype 1b, JFH-1 (AB047639) for genotype 2a, and HC-J8 (D10988) for genotype 2b.

\section{Evaluation of the Liver Functional Reserve}

The Child-Pugh classification was used to assess the liver functional reserve. In this study, the original version was used for the Child-Pugh 
classification [25], where ascites was graded as follows: $1=$ none, $2=$ mild, and $3=$ moderate. Moreover, the albumin-bilirubin (ALBI) grading system was adopted because it is known that liver functional reserve can be categorized in more detail compare to the Child-Pugh classification. The ALBI score was calculated based on serum albumin and total bilirubin values using the following formula: ALBI score $=[\log 10$ bilirubin $(\mu \mathrm{mol} / \mathrm{L}) \times 0.66$ ] + [albumin $(\mathrm{g} /$ L) $\times-0.085]$. The ALBI grade was defined by the following scores: $\leq-2.60$ for grade 1 , between -2.60 and -1.39 for grade 2 , and $>$ -1.39 for grade 3 . Furthermore, the middle ALBI grade (grade 2) was subdivided into 2a and $2 \mathrm{~b}$ [designated as the modified ALBI (mALBI) grade] by an ALBI score of -2.270 , corresponding to the cutoff value for a 15-min indocyanine green retention rate of 30\% [26].

\section{Statistical Analyses}

Continuous variables are presented as medians and ranges in parentheses. Categorical variables are presented as numbers and percentages. The Wilcoxon signed-rank test was used to evaluate the time-course changes in serum albumin, total bilirubin, eGFR, prothrombin time, Child-Pugh class/score, and ALBI grade/score. The Fisher's exact test, chi-square test, and Tukey's multiple comparison test were used to compare groups, as appropriate. The Cochran-Armitage trend test was used to evaluate associations between a variable with two categories and a variable with multiple categories. A $p$ value of $<0.05$ was considered statistically significant. All statistical analyses were performed using IBM SPSS Statistics v.26.0 (IBM Japan, Tokyo, Japan) and JMP v.12.0.1 (SAS Institute, Cary, NC, USA).

\section{RESULTS}

\section{Patient Characteristics}

This study involved 71 patients with decompensated cirrhosis, comprising 38 males and 33 females, with a median age of 65 (43-86) years (Table 1). Fifty-two and 19 patients were
Table 1 Baseline characteristics of the 71 patients

\begin{tabular}{|c|c|}
\hline Variable & $N=71$ \\
\hline Gender (male/female) & $38 / 33$ \\
\hline Age (years) & $65(43-86)$ \\
\hline White blood cell $\left(/ \mathrm{mm}^{3}\right)$ & $3420(1500-7800)$ \\
\hline Hemoglobin $(\mathrm{g} / \mathrm{dL})$ & $11.7(7.9-16.0)$ \\
\hline Platelets $\left(\times 10^{3} / \mathrm{mm}^{3}\right)$ & $77(29-186)$ \\
\hline AST (U/L) & $55(20-152)$ \\
\hline $\operatorname{ALT}(\mathrm{U} / \mathrm{L})$ & $31(11-119)$ \\
\hline Serum albumin $(\mathrm{g} / \mathrm{dL})$ & $2.9(1.9-4.5)$ \\
\hline Total bilirubin (mg/dL) & $1.5(0.5-5.2)$ \\
\hline Prothrombin time $(\%)$ & $64.0(18.0-110.0)$ \\
\hline Child-Pugh score & $8(5-13)$ \\
\hline Child-Pugh class $(\mathrm{A} / \mathrm{B} / \mathrm{C})$ & $7 / 47 / 17$ \\
\hline ALBI score & $\begin{array}{l}-1.58(-3.01 \text { to } \\
-0.45)\end{array}$ \\
\hline ALBI grade $(1 / 2 a / 2 b / 3)$ & $1 / 4 / 37 / 29$ \\
\hline $\begin{array}{l}\text { Ascites (none/mild/moderate or } \\
\text { severe) }\end{array}$ & $35 / 30 / 6$ \\
\hline $\begin{array}{l}\text { Hepatic encephalopathy (absent/ } \\
\text { mild) }\end{array}$ & $48 / 23$ \\
\hline Administration of BCAA (no/yes) & $31 / 40$ \\
\hline $\begin{array}{l}\text { Administration of diuretics (no/ } \\
\text { yes) }\end{array}$ & $23 / 48$ \\
\hline $\begin{array}{l}\text { Esophageal varices (absent / } \\
\text { present/unknown) }\end{array}$ & $20 / 45 / 6$ \\
\hline Alpha-fetoprotein $(\mathrm{ng} / \mathrm{mL})$ & $7.0(1.3-2031.8)$ \\
\hline HCV RNA $(\log \mathrm{IU} / \mathrm{mL})$ & $5.7(3.0-7.4)$ \\
\hline $\mathrm{HCV}$ genotype $(1 \mathrm{~b} / 2 \mathrm{a} / 2 \mathrm{~b})$ & $52 / 17 / 2$ \\
\hline $\begin{array}{l}\text { History of IFN-free DAAs } \\
\text { (naïve/experienced) }\end{array}$ & $70 / 1$ \\
\hline $\begin{array}{l}\text { History of HCC treatment (no/ } \\
\text { yes) }\end{array}$ & $49 / 22$ \\
\hline
\end{tabular}

$A S T$ aspartate aminotransferase; $A L T$ alanine aminotransferase; $B C A A$ branched chain amino acid; $H C V$ hepatitis $\mathrm{C}$ virus; IFN interferon; $D A A s$ direct-acting antivirals; $H C C$ hepatocellular carcinoma 
infected with genotype 1 and 2, respectively. The median values of albumin, total bilirubin, and prothrombin time were $2.9(1.9-4.5) \mathrm{g} / \mathrm{dL}$, $1.5(0.5-5.2) \mathrm{mg} / \mathrm{dL}$, and 64.0 (18.0-110.0) \%, respectively. The median Child-Pugh score was 8 (5-13) points. Seven patients were Child-Pugh class A (score 5-6), 47 were class B (score 7-9), and 17 were class $C$ (score 10-15). All the patients with Child-Pugh class A had previous decompensated events, and their Child-Pugh scores improved after branched-chain amino acid (BCAA) and/or diuretics administration; consequently, the Child-Pugh class improved to $\mathrm{A}$ at the initiation of SOF/VEL. ALBI grades 1 , $2 \mathrm{a}, 2 \mathrm{~b}$, and 3 were registered in $1,4,37$, and 29 patients, respectively. Among the 71 patients, $45(63.4 \%)$ had esophageal varices, and 12 were treated by an endoscopic procedure before SOF/ VEL initiation. Twenty-two (31.0\%) patients underwent previous treatment for HCC (radiofrequency ablation in 9 patients, surgical resection in 3, transcatheter arterial chemoembolization in 5 , chemotherapy in 1 , heavy ion therapy in 1, and proton therapy 1 ; all performed 2 months to 13 years before the entry into this study). None of the patients had evidence of HCC on imaging modalities at the initiation of SOF/VEL. Forty (56.3\%) patients were administered BCAA for hypoalbuminemia and hepatic encephalopathy, and 48 (67.6\%) patients were administered diuretics (furosemide, spironolactone, and/or tolvaptan) for hepatic edema. Other clinical characteristics at the initiation of SOF/VEL are shown in Table 1.

\section{Virologic Responses}

Among the 71 patients, 69 completed the treatment as scheduled; the remaining 2 patients prematurely discontinued treatment due to AEs (as described below). The overall SVR12 rate, based on intention-to-treat analysis, was $94.4 \%$ (67/71; Fig. 1a). Four patients did not achieve SVR12-two relapsed, and one discontinued treatment at week 9 (when serum HCV RNA was undetectable) due to acute cholecystitis and died of sepsis 3 weeks later; thus, the final virologic response was undetermined. The final patient that did not achieve SVR12 completed the treatment and achieved SVR4, but developed spontaneous bacterial peritonitis and died of liver failure at week 10 after the completion of treatment. The two deaths exhibited no viremia during treatment; thus, all the 71 patients achieved EOT. According to the Child-Pugh classification, the SVR12 rates were $85.7 \%(6 / 7)$ for class A, 97.9\% (46/47) for class B, and $88.2 \%$ (15/17) for class C (Fig. 1b). The SVR12 patients with Child-Pugh class $C$ included one individual with a Child-Pugh score of 13 , who would have been excluded from the Japanese phase 3 trial [21]. According to the ALBI grading system, the SVR12 rates were $0 \%(0 / 1)$ for grade $1,100 \%(4 / 4)$ for grade $2 a, 97.3 \%(36 / 37)$ for grade $2 b$, and $93.1 \%(27 / 29)$ for grade 3 (Fig. 1c). Among the 22 patients with HCC treatment history, 20 (90.9\%) achieved SVR12 (Fig. 1d), while the remaining 2 patients relapsed (both were naïve to DAA) (Table S1). Of the 20 patients with SVR12, 4 developed recurrent HCC during the follow-up period: 1 underwent radiofrequency ablation for solitary lesion, 1 underwent CyberKnife treatment for solitary lesion, 1 underwent hepatic arterial infusion chemotherapy using a reservoir for portal vein thrombosis, and 1 is awaiting liver transplantation due to deteriorated liver function despite a solitary lesion. Sixty-six of the 70 DAA-naïve patients (94.3\%) achieved SVR12, while 1 DAA-experienced patient (prior treatment: asunaprevir plus daclatasvir) achieved SVR12.

We sequentially analyzed the resistance-associated substitutions (RASs) in the NS5A region of the two patients who relapsed. In one patient (genotype 1b), no RASs were detected at position L31, Q54, and Y93 at baseline; however, L31V, F37L, and Y93N emerged at relapse. In the other patient (genotype 2b), Q30K and L31M had already existed at baseline; thereafter, both Q30K and L31M remained at relapse. Neither relapser had NS5B RASs (including S282T) at baseline or relapse.

\section{Time-Course Changes in the Liver Functional Reserve}

Changes in the biochemical and liver functional reserve parameters from baseline to 12 weeks 


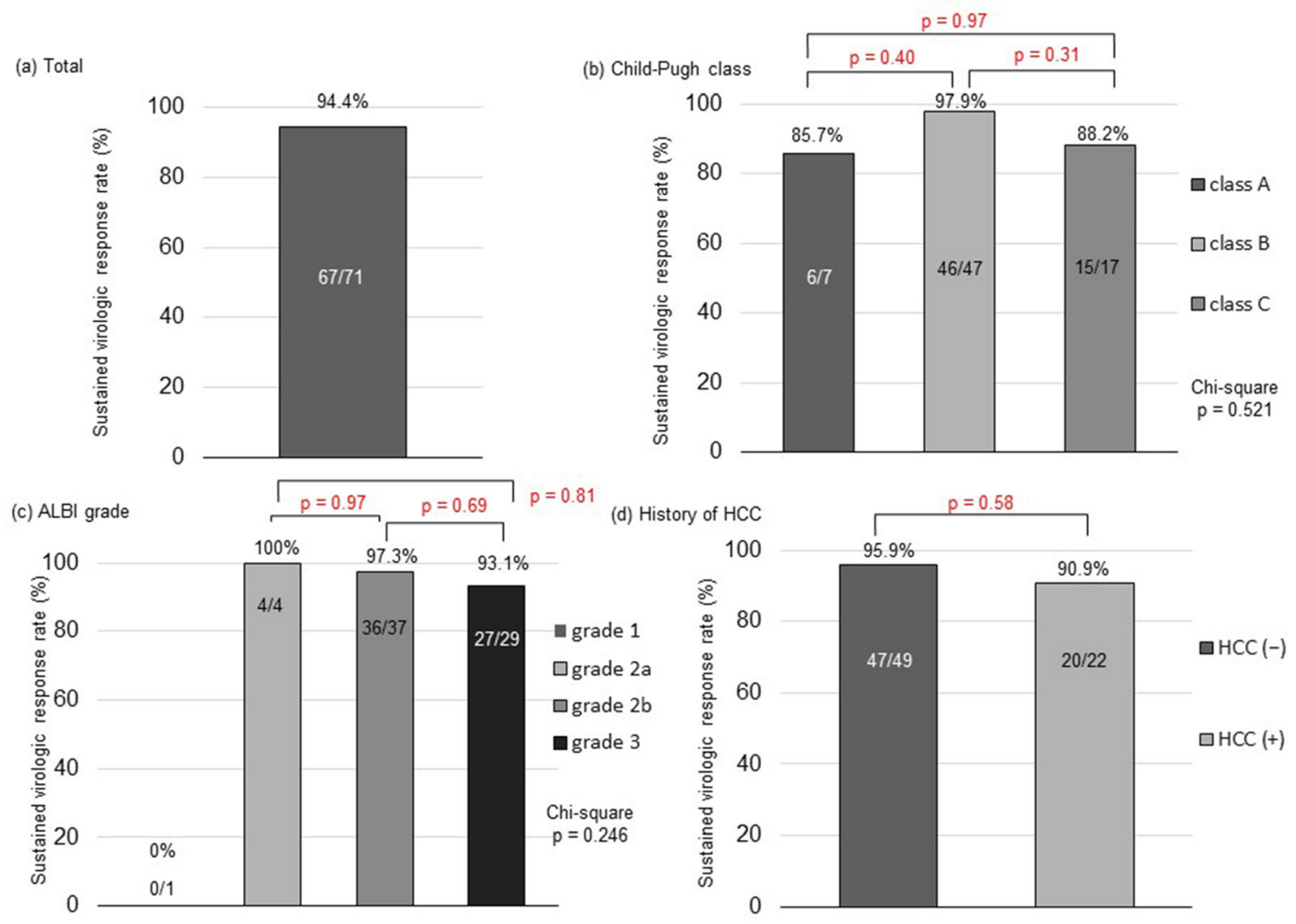

Fig. 1 Sustained virologic response rates according to each category. a A sustained virologic response rate in a total of 71 patients with decompensated liver cirrhosis based on intention-to-treat analysis. b Comparison of sustained virologic response rates among patients with Child-Pugh

post-treatment were analyzed in the 67 patients who achieved SVR12. The median total bilirubin levels did not change $(p=0.09$; Fig. $2 \mathrm{a})$. The median prothrombin time significantly improved from 64.0 to $71.0 \%$ ( $p=0.001$; Fig. $2 b)$. The median albumin levels significantly increased from 2.9 to $3.3 \mathrm{~g} / \mathrm{dL}$ ( $p<0.001$; Fig. $2 \mathrm{c}$ ).

The median Child-Pugh score significantly improved, from 8 points at baseline to 7 points at 12 weeks post-treatment ( $p<0.001$; Fig. $2 d)$. The proportion of Child-Pugh class A patients increased from 9.0 to $37.3 \%$, while that of classes B and C patients decreased from 68.6 to $49.3 \%$ and from 22.4 to $13.4 \%$, respectively. Accordingly, the liver functional reserve based on the Child-Pugh classification significantly improved in the patients who achieved SVR12

classes A, B, and C. c Comparison of sustained virologic response rates among patients with ALBI grades $1,2 \mathrm{a}, 2 \mathrm{~b}$, and 3. $\mathbf{d}$ Comparison of sustained virologic response rates between patients with and without a history of hepatocellular carcinoma $(H C C)$

$(p<0.001$; Fig. 3a). Among the 17 class C patients, 4 improved to class $\mathrm{B}$ and 2 improved to class A. Among the 47 class B patients, 17 improved to class A (Fig. S1). No patients showed a deterioration in Child-Pugh class, but three patients exhibited worse scores after achieving SVR12 (i.e., 7-8, 8-9, and 11-12).

The median ALBI grade scores also improved from -1.59 at baseline to -1.90 at 12 weeks post-treatment $(p<0.001 ;$ Fig. $2 \mathrm{e})$. Grade 1 increased from 0.0 to $7.5 \%$, grade $2 \mathrm{a}$ increased from 6.0 to $14.9 \%$, and grade $2 b$ increased from 53.7 to $59.7 \%$. In contrast, grade 3 decreased from 40.3 to $17.9 \%$. Accordingly, the liver functional reserve based on the mALBI grade also significantly improved in patients who achieved SVR12 ( $p<0.001 ;$ Fig. 3b). Among the 



Fig. 2 Time-course changes in the biochemical and hepatic functional reserve parameters. Time-course changes from baseline to 12-week post-treatment in a total bilirubin levels, b prothrombin time, c serum albumin

29 grade 3 patients, 15 improved to grade $2 b$ and one improved to grade $2 \mathrm{a}$. Among the 37 grade $2 \mathrm{~b}$ patients, e8ight improved to grade $2 \mathrm{a}$ and 3 improved to grade 1 . Two patients with grade $2 \mathrm{a}$ improved to grade 1 . However, two patients deteriorated mALBI grades despite achieving SVR12: one from grade $2 b$ to grade 3 and the other from grade $2 \mathrm{a}$ to grade $2 \mathrm{~b}$ (Fig. S2). Both patients were administered tolvaptan for refractory ascites, and the former underwent a transjugular intrahepatic portosystemic shunt procedure due to non-responsiveness to tolvaptan.

Taken together, improvements in these surrogate parameters indicate that the achievement of SVR12 with SOF/VEL could restore the liver function reserve during the short term of 24 weeks in patients with decompensated cirrhosis. However, the ALBI grade and score deteriorated in some patients with refractory ascites, despite achieving SVR12.

\section{Time-Course Changes in Drug Administration for Complications in Patients with Decompensated Cirrhosis}

Given that the serum albumin levels, Child-Pugh scores/classes, and ALBI grades/scores improved in patients who achieved SVR12, we investigated if the diuretic doses and BCAAs were reduced or discontinued with SVR12. Among the 67 patients who achieved SVR12, 45 (67.2\%) had been administered diuretics and 22 (32.8\%) were diuretics free at baseline. Of the 45 patients receiving diuretics, 13 discontinued 


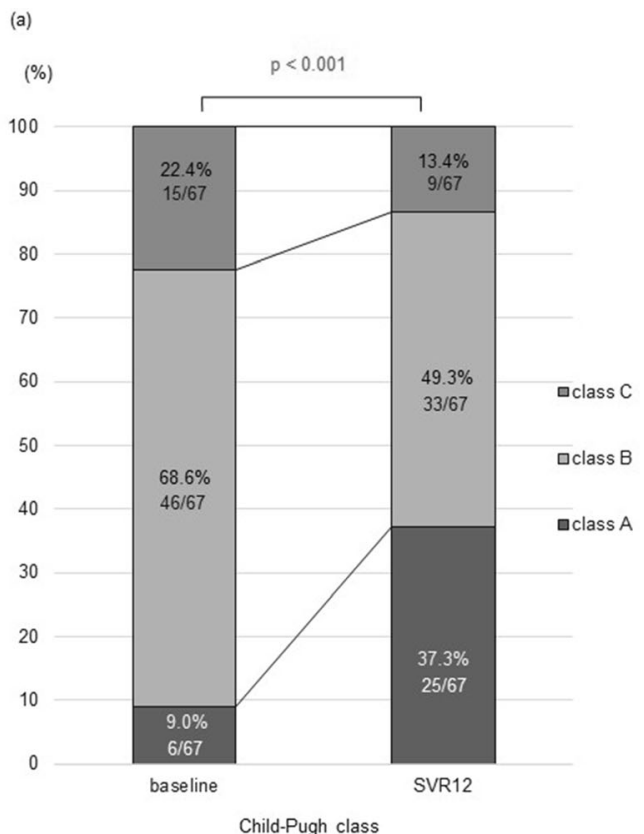

Fig. 3 Time-course changes in the distribution of the Child-Pugh class and ALBI grade. a Child-Pugh class and b ALBI grade significantly improved from baseline to 12-week post-treatment in patients who achieved sustained

diuretic use and 2 reduced their doses with the achievement of SVR12. Twenty-five patients continued the same dosages, and five patients increased their doses. One patient initiated diuretic treatment despite achieving SVR12. Thus, the proportion of patients who maintained, increased, or initiated diuretic use during the study period significantly decreased to $46.3 \%(31 / 67)$ at 12 weeks post-treatment $(p=0.01$; Fig. $4 \mathrm{a})$. The proportion of patients receiving BCAA treatment significantly decreased from $53.7 \%(36 / 67)$ at baseline to $37.3 \%(25 / 67)$ at 12 weeks post-treatment. Thus, 11 of the 36 patients receiving BCAAs spared BCAAs $(p=0.02$; Fig. $4 \mathrm{~b})$. These results suggest that achieving SVR12 with SOF/VEL could improve the severity of cirrhosis-related complications, thereby reducing or sparing drug administration dosages for the treatment of complications in patients with decompensated cirrhosis. Such results have not been reported before. (b)

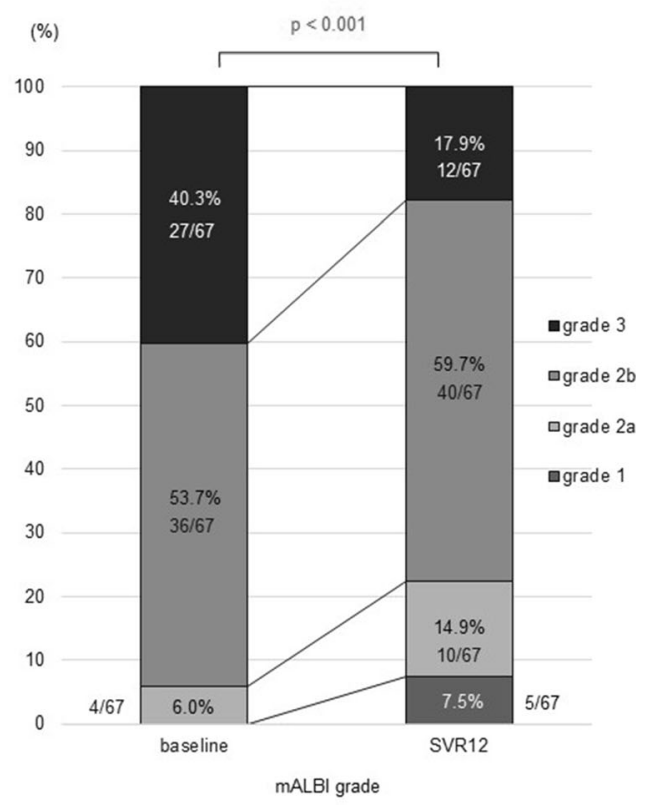

virologic response at 12 weeks post-treatment (SVR12) $\left(p=7.19 \times 10^{-4}\right.$ and $2.42 \times 10^{-4}$, respectively, by the Cochran-Armitage trend test)

\section{Adverse Events}

Overall, AEs were observed during the treatment and follow-up periods in $19.7 \%(14 / 71)$ of the patients. The incidence was higher in the Child-Pugh class C group [35.3\% (6/17)] than in the Child-Pugh class A/B group $[14.8 \%(8 /$ 54)], although not statistically significant $(p=0.08$; Table 2). Serious AEs occurred more frequently in the former $[11.8 \%(2 / 17)]$ than in the latter $[3.8 \%(2 / 54)]$, although not statistically significant $(p=0.241)$. AEs leading to treatment discontinuation were observed in two patients with Child-Pugh class B: acute cholecystitis in one (as described above) and esophageal varices rupture in the other (as described below). Two patients died during the follow-up period (as described above): one Child-Pugh class B patient had diabetes mellitus and cholecystolithiasis at baseline and developed acute cholecystitis and later died of sepsis; and the other Child-Pugh class C patients had total bilirubin and albumin levels that fluctuated at $3.2-3.8 \mathrm{mg} / \mathrm{dL}$ and $2.3-3.0 \mathrm{~g} /$ 


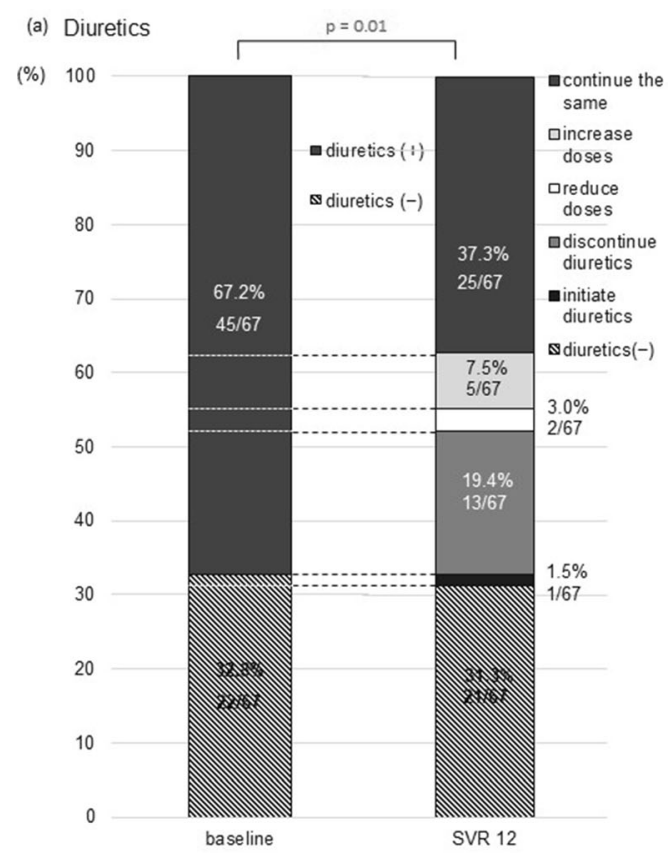

Fig. 4 Time-course changes in the administration dosages of drugs for the treatment of complications. a Changes in administration doses of diuretics for hepatic edema. The proportion of patients who received the same, increased the doses or initiated diuretics significantly decreased after achieving sustained virologic response at 12 -week posttreatment (SVR12) $\quad\left(p=1.46 \times 10^{-2} \quad\right.$ by the Cochran-Armitage trend test). b Changes in

$\mathrm{dL}$, respectively, during the treatment period and developed spontaneous bacterial peritonitis and consequently died of liver failure. Two patients with Child-Pugh class B and ALBI grade 3 developed encephalopathy grade 2 during the treatment period, but rapidly recovered with hydration and achieved SVR12 after treatment completion. After achieving SVR12, both patients improved the liver functional reserve: Child-Pugh B to A in one and ALBI grade 3 to $2 \mathrm{~b}$ in both. Two patients developed variceal bleeding during the treatment period, but both achieved SVR12: one patient showed varices grade 3 with red signs at baseline and discontinued treatment at week 8 (as described above); and the other patient exhibited varices grade 2 without red signs at baseline and completed the treatment. Neither patient underwent endoscopic treatment before SOF/VEL, albeit the 12 patients who had undergone

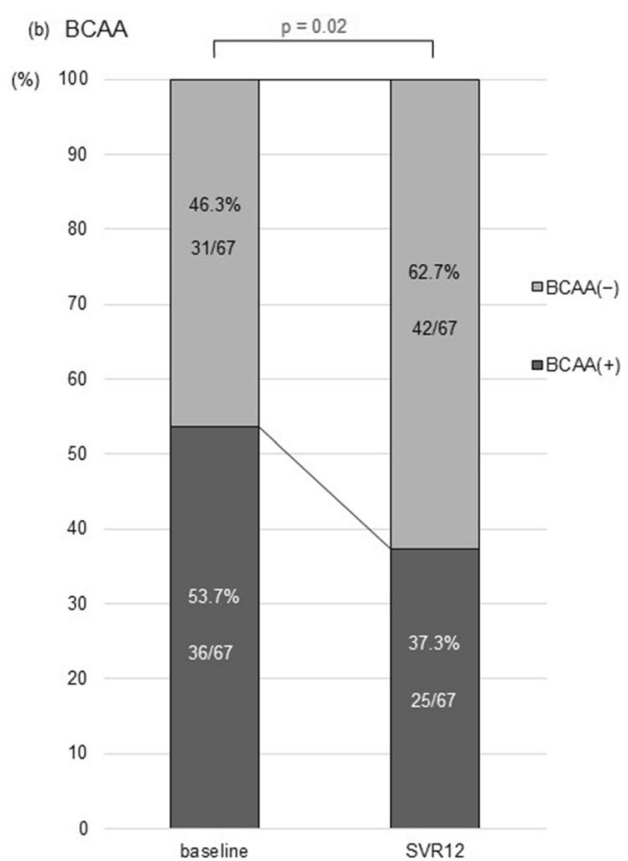

administration of branched chain amino acid (BCAA) for hepatic encephalopathy and/or hypoalbuminemia. The proportion of patients who continued to receive BCAA treatment was significantly reduced from baseline to 12 -week post-treatment among patients who achieved SVR12 $\left(p=2.44 \times 10^{-2}\right.$ by the Cochrane-Armitage trend test)

endoscopic treatment for esophageal varices did not develop variceal rupture. No patients had elevated transaminase, but one patient experienced increased total bilirubin from 0.5 to $2.3 \mathrm{mg} / \mathrm{dL}$ (grade 1). The median eGFR levels did not vary from the baseline to 12 weeks posttreatment $\left(70.0 \mathrm{~mL} / \mathrm{min} / 1.73 \mathrm{~m}^{2}\right.$ for both; $p=0.11$; Fig. S3).

\section{DISCUSSION}

To date, there have been only two phase 3 clinical trials of SOF/VEL with or without ribavirin for patients with decompensated cirrhosis: one from the United States (ASTRAL-4) for those with Child-Pugh class B (score of 7-9) [13]; and the other from Japan for those with Child-Pugh score $\leq 12$ [21]. Our prospective, real-world, multicenter study included patients 
Table 2 Adverse events

\begin{tabular}{|c|c|c|c|c|}
\hline Adverse events & $\begin{array}{l}\text { Total } \\
(n=71)\end{array}$ & $\begin{array}{l}\text { Child-Pugh class } A \text { or } B \\
(n=54)\end{array}$ & $\begin{array}{l}\text { Child-Pugh class C } \\
(n=17)\end{array}$ & $p$ value \\
\hline Any adverse events & $14(19.7 \%)$ & $8(14.8 \%)$ & $6(35.3 \%)$ & 0.08 \\
\hline Death & $2(2.8 \%)$ & $1(1.9 \%)$ & $1(5.9 \%)$ & 0.42 \\
\hline $\begin{array}{l}\text { Serious adverse events (CTCAE } \\
\text { grade } \geq 4 \text { ) }\end{array}$ & $4(5.6 \%)$ & $2(3.8 \%)$ & $2(11.8 \%)$ & 0.24 \\
\hline $\begin{array}{l}\text { Adverse events leading to treatment } \\
\text { discontinuation }\end{array}$ & $2(2.8 \%)$ & $2(3.8 \%)$ & $0(0.0 \%)$ & 1.00 \\
\hline \multicolumn{5}{|l|}{ CTCAE grade } \\
\hline Grade 1 & $5(7.0 \%)$ & $3(5.7 \%)$ & $2(11.8 \%)$ & 0.59 \\
\hline Grade 2 & $2(2.8 \%)$ & $1(1.9 \%)$ & $1(5.9 \%)$ & 0.42 \\
\hline Grade 3 & $3(4.2 \%)$ & $2(3.8 \%)$ & $1(5.9 \%)$ & 0.57 \\
\hline Grade 4 & $2(2.8 \%)$ & $1(1.9 \%)$ & $1(5.9 \%)$ & 0.42 \\
\hline Grade 5 & $2(2.8 \%)$ & $1(1.9 \%)$ & $1(5.9 \%)$ & 0.42 \\
\hline \multicolumn{5}{|l|}{ Adverse events } \\
\hline Nosebleed & $1(1.4 \%)$ & $0(0.0 \%)$ & $1(5.9 \%)$ & 0.24 \\
\hline Dysgeusia & $1(1.4 \%)$ & $1(1.9 \%)$ & $0(0.0 \%)$ & 1.00 \\
\hline Hepatic encephalopathy & $2(2.8 \%)$ & $2(3.8 \%)$ & $0(0.0 \%)$ & 1.00 \\
\hline Progression of diabetes mellitus & $1(1.4 \%)$ & $0(0.0 \%)$ & $1(5.9 \%)$ & 0.24 \\
\hline Esophageal varices rupture & $2(2.8 \%)$ & $1(1.9 \%)$ & $1(5.9 \%)$ & 0.42 \\
\hline Acute cholecystitis & $1(1.4 \%)$ & $1(1.9 \%)$ & $0(0.0 \%)$ & 1.00 \\
\hline Deterioration of ascites & $2(2.8 \%)$ & $1(1.9 \%)$ & $1(5.9 \%)$ & 0.42 \\
\hline Fatigue & $1(1.4 \%)$ & $0(0.0 \%)$ & $1(5.9 \%)$ & 0.24 \\
\hline Pharynx discharge & $1(1.4 \%)$ & $1(1.9 \%)$ & $0(0.0 \%)$ & 1.00 \\
\hline Liver failure & $1(1.4 \%)$ & $0(0.0 \%)$ & $1(5.9 \%)$ & 0.24 \\
\hline \multicolumn{5}{|l|}{ Laboratory abnormalities } \\
\hline Total bilirubin elevation & $1(1.4 \%)$ & $1(1.9 \%)$ & $0(0.0 \%)$ & 1.00 \\
\hline
\end{tabular}

Adverse events were graded according to the Common Terminology Criteria for Adverse Events (CTCAE) v.4.0, as presented by the National Cancer Institute Cancer Therapy Evaluation Program

with more advanced disease stages or unfavorable conditions; however, it achieved high effectiveness, acceptable safety profiles, and good tolerability of 12-week SOF/VEL in decompensated cirrhotic patients with genotypes 1 and 2 (Child-Pugh score $\leq 13$ ) in real- world clinical practice. The SVR rate of $94.4 \%$ $(67 / 71)$ in our study is similar to those of 12-week SOF/VEL without ribavirin in the ASTRAL-4 study [88.8\% (16/18) for genotype $1 \mathrm{~b}$ and 100\% (4/4) for genotype 2] and in the Japan phase 3 study $(92.2 \%$; 47/51) [21]. Real-world 
study cohorts may include patients with more unfavorable factors or conditions, unlike the participants in phase 3 clinical trials. For instance, our study cohort included one patient with a Child-Pugh score of 13 , who would have been ineligible for the previous phase 3 studies $[13,21]$. Nevertheless, this individual completed the treatment safely and improved to score 12 after achieving SVR. Likewise, $31.0 \%$ of the patients had undergone prior treatment modalities for HCC, and $91.3 \%$ of those patients achieved SVR after treatment completion (some of whom would have been ineligible for the above studies $[13,21]$. In many patients, the liver functional reserve was restored in the short period, suggesting that the achievement of SVR might provide more treatment options for recurrent HCC. However, deteriorated liver functional reserve was observed in some patients despite achieving SVR; hence, a better understanding of the limitations of the treatment (e.g., identification of such patients) is required.

Previous and our studies have indicated that the achievement of SVR with 12-week SOF/VEL could retrieve the liver functional reserve over a short time of 24 weeks even for patients with decompensated cirrhosis [21]. Notably, we suggest that the restored liver functional reserve could improve cirrhosis-related complications and consequently reduce or spare the doses of drug, such as diuretics for hepatic edema and BCAA preparations for hepatic encephalopathy and hypoalbuminemia. This is the first report to clarify such beneficial effects of VEL/SOF on complications and related treatments. Given our previous report on the poor prognosis of cirrhotic patients receiving high-dose diuretics for hepatic edema [27], reduced or spared diuretic use may lead to improvements in the mid- and long-term prognosis of patients with decompensated cirrhosis, especially those with hepatic edema.

An advantage of the SOF/VEL regimen is that a protease inhibitor, which may cause hepatopathy, is not included. We also observed no increases in the transaminase levels in this study. Increased total bilirubin was observed in one patient, but the relationship between this result and the drugs is unclear; perhaps, the treatment was administered during naturallyoccurring deterioration.

In our real-world cohort, treatment was prematurely discontinued due to cholecystitis and esophageal varices rupture. Specifically, patients with advanced decompensated cirrhosis may be vulnerable to acute infection, which can be difficult to treat or control. Uncontrollable or rapidly progressive infections could impair the functioning of the liver and other organs, resulting in multiple organ failure. In our study, acute cholecystitis followed by sepsis and spontaneous bacterial peritonitis caused or triggered patient deaths. Therefore, physicians and patients must pay scrupulous attention to $\mathrm{HCV}$ and acute infections. The Japanese phase 3 study also reported esophageal varices rupture during treatment [21]; in our study, the two patients with variceal rupture had esophageal varices $\geq$ grade 2 with or without red signs and had not undergone endoscopic treatment before SOF/VEL. These results suggest that advanced treatment for esophageal varices should be considered for such patients prior to SOF/VEL. Our results indicate no association between SOF/VEL and acute infections or esophageal varices rupture.

The current study has some limitations. First, the sample size was small, although it did exceed the sample size of previous phase 3 studies [13, 21]. Second, this was an open-label study. Third, the mid- and long-term effects of achieving SVR with SOF/VEL on the liver functional reserve, health-related quality of life, and prognosis remain unclear. Finally, the RASs at baseline and relapse were analyzed in patients with virologic failure, but the pre-existing RASs to SOF/VEL were not analyzed in all the patients.

\section{CONCLUSION}

This prospective, multicenter study demonstrated the high efficacy, acceptable safety, and good tolerability of 12-week SOF/VEL in decompensated cirrhotic patients with genotypes 1 and 2 in real-world clinical practice. The achievement of SVR not only restored the liver functional reserve but also reduced or spared 
the use of diuretics and BCAA preparations for the treatment of complications over a short term of 24 weeks.

\section{ACKNOWLEDGEMENTS}

We thank the participants of the study.

KTK49 Liver Study Group Members: Masanori Atsukawa, Akihito Tsubota, Chisa Kondo, Hidenori Toyoda, Makoto Nakamuta, Koichi Takaguchi, Tsunamasa Watanabe, Atsushi Hiraoka, Haruki Uojima, Toru Ishikawa, Motoh Iwasa, Toshifumi Tada, Akito Nozaki, Makoto Chuma, Shinya Fukunishi, Akira Asai, Toru Asano, Chikara Ogawa, Hiroshi Abe, Naoki Hotta, Toshihide Shima, Etsuko Iio, Shigeru Mikami, Yoshihiko Tachi, Shinichi Fujioka, Hironao Okubo, Noritomo Shimada, Joji Tani, Isao Hidaka, Akio Moriya, Kunihiko Tsuji, Takehiro Akahane, Naoki Yamashita, Tomomi Okubo, Taeang Arai, Kiyoshi Morita, Kazuhito Kawata, Yasuhito Tanaka, Takeshi Okanoue, Shin Maeda, Takashi Kumada, Katsuhiko Iwakiri, Akemi Tsutsui, Notio Itokawa, Keizo Kato, Tsunekazu Oikawa, Isao Sakaida, Asahiro Morishita.

Funding. No funding or sponsorship was received for this study or publication of this article.

Authorship. All named authors meet the International Committee of Medical Journal Editors (ICMJE) criteria for authorship for this article, take responsibility for the integrity of the work as a whole, and have given their approval for this version to be published.

Authorship Contributions. MA, AT, HT, KT, TM, NM and SM contributed to the concept of this study. MA and AT equally contributed to manuscript writing. CK and AT performed the statistical data analyses. MA, AT, HT, KT, TW, AH, HU, TI, MI, TT, AK, MC, SF, AA, CO, HA, TS, EI, SM, YT, SF, HO, NS, JT, IH, AM, KT, TA, NY, TO, TA, KM, KK, YT, YO, SM and KI contributed to patient's recruitment, enrollment, clinical assessments, and data collection. All authors approved the final manuscript.

Disclosures. Masanori Atsukawa, Hidenori Toyoda, Koichi Takaguchi and Takashi Kumada received lecture fees from Gilead Sciences. Akihito Tsubota, Chisa Kondo, Makoto Nakamuta, Tsunamasa Watanabe, Atsushi Hiraoka, Haruki Uojima, Toru Ishikawa, Motoh Iwasa, Toshifumi Tada, Akito Nozaki, Makoto Chuma, Shinya Fukunishi, Akira Asai, Toru Asano, Chikara Ogawa, Hiroshi Abe, Naoki Hotta, Toshihide Shima, Etsuko Iio, Shigeru Mikami, Yoshihiko Tachi, Shinichi Fujioka, Hironao Okubo, Noritomo Shimada, Joji Tani, Isao Hidaka, Akio Moriya, Kunihiko Tsuji, Takehiro Akahane, Naoki Yamashita, Tomomi Okubo, Taeang Arai, Kiyoshi Morita, Kazuhito Kawata, Yasuhito Tanaka, Takeshi Okanoue, Shin Maeda and Katsuhiko Iwakiri have nothing to disclose.

Compliance with Ethics Guidelines. This study was approved by the ethics committee of Nippon Medical School (IRB \#R1-05-1129. All documents approved by Nippon Medical School (Atsukawa M) were sent to each institution and approved by each institution under the same IRB name. This study was performed in accordance with the 2013 Helsinki Declaration. All patients consented to provide their data. Written informed consent was obtained from each patient before enrollment.

Data Availability. The datasets are available from the corresponding author on reasonable request.

Open Access. This article is licensed under a Creative Commons Attribution-NonCommercial 4.0 International License, which permits any non-commercial use, sharing, adaptation, distribution and reproduction in any medium or format, as long as you give appropriate credit to the original author(s) and the source, provide a link to the Creative Commons licence, and indicate if changes were made. The images or other third party material in this article are included in the article's Creative Commons licence, unless indicated otherwise in a credit line to the material. If material is not included 
in the article's Creative Commons licence and your intended use is not permitted by statutory regulation or exceeds the permitted use, you will need to obtain permission directly from the copyright holder. To view a copy of this licence, visit http://creativecommons.org/licenses/by$\mathrm{nc} / 4.0 /$.

\section{REFERENCES}

1. D'Amico G, Garcia-Tsao G, Pagliaro L. Natural history and prognostic indicators of survival in cirrhosis: a systematic review of 118 studies. J Hepatol. 2006;44(1):217-31.

2. Mar J, Martínez-Baz I, Ibarrondo O, et al. Survival and clinical events related to end-stage liver disease associated with $\mathrm{HCV}$ prior to the era of all oral direct-acting antiviral treatments. Expert Rev Gastroenterol Hepatol. 2019;13(7):699-708.

3. Okubo T, Atsukawa M, Tsubota A, et al. Efficacy and safety of ledipasvir/sofosbuvir for genotype $1 \mathrm{~b}$ chronic hepatitis $\mathrm{C}$ patients with moderate renal impairment. Hepatol Int. 2018;12(2):133-42.

4. Arai T, Atsukawa M, Tsubota A, et al. Efficacy and safety of ombitasvir/paritaprevir/ritonavir combination therapy for genotype $1 \mathrm{~b}$ chronic hepatitis $\mathrm{C}$ patients complicated with chronic kidney disease. Hepatol Res. 2018;48(7):549-55.

5. Atsukawa M, Tsubota A, Toyoda H, et al. Efficacy and safety of ombitasvir/paritaprevir/ritonavir and ribavirin for chronic hepatitis patients infected with genotype 2a in Japan. Hepatol Res. 2019;49(4): 369-76.

6. Atsukawa M, Tsubota A, Toyoda H, et al. Efficacy and safety of elbasvir/grazoprevir for Japanese patients with genotype $1 \mathrm{~b}$ chronic hepatitis $\mathrm{C}$ complicated by chronic kidney disease, including those undergoing hemodialysis: a post hoc analysis of a multicenter study. J Gastroenterol Hepatol. 2019;34(2):364-9.

7. Atsukawa M, Tsubota A, Toyoda H, et al. The efficacy and safety of glecaprevir plus pibrentasvir in 141 patients with severe renal impairment: a prospective, multicenter study. Aliment Pharmacol Ther. 2019;49(9):1230-41.

8. Toyoda H, Atsukawa M, Watanabe $\mathrm{T}$, et al. Realworld experience of 12-week direct-acting antiviral regimen of glecaprevir and pibrentasvir in patients with chronic hepatitis $\mathrm{C}$ virus infection. J Gastroenterol Hepatol. 2020;35(5):855-61.
9. Ikeda H, Watanabe T, Atsukawa M, et al. Evaluation of 8-week glecaprevir/pibrentasvir treatment in direct-acting antiviral-naïve noncirrhotic $\mathrm{HCV}$ genotype 1 and 2infected patients in a real-world setting in Japan. J Viral Hepat. 2019;26(11): 1266-75.

10. Nozaki A, Atsukawa M, Kondo C, KTK49 Liver Study Group, et al. The effectiveness and safety of glecaprevir/pibrentasvir in chronic hepatitis $\mathrm{C}$ patients with refractory factors in the real world: a comprehensive analysis of a prospective multicenter study. Hepatol Int. 2020;14(2):225-38.

11. Charlton M, Everson GT, Flamm SL, SOLAR-1 Investigators, et al. Ledipasvir and sofosbuvir plus ribavirin for treatment of $\mathrm{HCV}$ infection in patients with advanced liver disease. Gastroenterology. 2015;149(3):649-59.

12. Manns M, Samuel D, Gane EJ, SOLAR-2 investigators, et al. Ledipasvir and sofosbuvir plus ribavirin in patients with genotype 1 or 4 hepatitis $C$ virus infection and advanced liver disease: a multicentre, open-label, randomised, phase 2 trial. Lancet Infect Dis. 2016;16(6):685-97.

13. Curry MP, O'Leary JG, Bzowej N, ASTRAL-4 Investigators, et al. Sofosbuvir and velpatasvir for HCV in patients with decompensated cirrhosis. N Engl J Med. 2015;373(27):2618-28.

14. Foster GR, Irving WL, Cheung MC, HCV Research, $\mathrm{UK}$, et al. Impact of direct acting antiviral therapy in patients with chronic hepatitis $\mathrm{C}$ and decompensated cirrhosis. J Hepatol. 2016;64(6):1224-311.

15. Belli LS, Berenguer M, Cortesi PA, European Liver, and Intestine Association (ELITA), et al. Delisting of liver transplant candidates with chronic hepatitis $\mathrm{C}$ after viral eradication: a European study. J Hepatol. 2016;65(3):524-31.

16. Fernández Carrillo C, Lens S, Llop E, et al. Treatment of hepatitis $C$ virus infection in patients with cirrhosis and predictive value of model for endstage liver disease: analysis of data from the Hepa-C registry. Hepatology. 2017;65(6):1810-22.

17. McCaughan GW, Thwaites PA, Roberts SK, Australian Liver Association Clinical Research Network, et al. Sofosbuvir and daclatasvir therapy in patients with hepatitis C-related advanced decompensated liver disease $(\mathrm{MELD} \geq 15)$. Aliment Pharmacol Ther. 2018;47(3):401-11.

18. Kato K, Shimada N, Atsukawa M, et al. Single nucleotide polymorphisms associated with elevated alanine aminotransferase in patients receiving asunaprevir plus daclatasvir combination therapy for chronic hepatitis C. PLoS ONE. 2019;14(7): e0219022. 
19. EASL Recommendations on Treatment of Hepatitis C. European Association for the study of the liver. J Hepatol. 2018;69(2):461-511

20. Hepatitis C Guidance. Guidance: AASLD-IDSA recommendations for testing, managing, and treating adults infected with hepatitis $C$ virus. AASLD/IDSA HCV Guid Panel Hepatol. 2015;62(3):932-54.

21. Takehara T, Sakamoto N, Nishiguchi S, et al. Efficacy and safety of sofosbuvir-velpatasvir with or without ribavirin in $\mathrm{HCV}$-infected Japanese patients with decompensated cirrhosis: an openlabel phase 3 trial. J Gastroenterol. 2019;54(1): 87-95.

22. National Cancer Institute Division of Cancer Treatment \& Diagnosis. https://ctep.cancer.gov/ protocolDevelopment/electronic_applications/ctc. htm\#ctc_50. Accessed 27 Mar 2020.

23. Ohno O, Mizokami M, Wu RR, et al. New hepatitis $\mathrm{C}$ virus (HCV) genotyping system that allows for identification of HCV genotypes $1 \mathrm{a}, 1 \mathrm{~b}, 2 \mathrm{a}, 2 \mathrm{~b}, 3 \mathrm{a}$, 3b, 4, 5a, and 6a. J Clin Microbiol. 1997;35(1): 201-7.

24. Dvory-Sobol H, Han B, Lu J, et al. In vitro resistance profile of hepatitis $C$ virus NS5A inhibitor velpatasvir in genotypes 1 to 6 . J Viral Hepat. 2019;26(8):991-1001.

25. Pugh RN, Murray-Lyon IM, Dawson JL, et al. Transection of the oesophagus for bleeding oesophageal varices. Br J Surg. 1973;60(8):646-9.

26. Hiraoka A, Michitaka K, Kumada T, et al. Validation and potential of albumin-bilirubin grade and prognostication in a nationwide survey of 46,681 hepatocellular carcinoma patients in Japan: the need for a more detailed evaluation of hepatic function. Liver Cancer. 2017;6(4):325-36.

27. Atsukawa M, Tsubota A, Takaguchi K, et al. Analysis of factors associated with the prognosis of cirrhotic patients who were treated with tolvaptan for hepatic edema. J Gastroenterol Hepatol. 2020;35(7): 1229-37. 